\title{
Green synthesis of carbon nanotubes impregnated with metallic nanoparticles: Characterization and application in glyphosate adsorption
}

DOI: https://doi.org/10.1016/i.chemosphere.2021.131193

\section{Authors}

Júlia C. Diel, Dison S.P. Franco, Andrei V. Igansi, Tito R.S. Cadaval Jr., Hércules A. Pereira, Isaac dos S. Nunes, Charles W. Basso, Maria do Carmo M. Alves, Jonder Morais, Diana Pinto, Guilherme L. Dotto

\begin{abstract}
In the present work, multi-walled carbon nanotubes (MWCNTs) were used as support material for the impregnation of metallic nanoparticles (MNPs) produced by green synthesis. The influences of the plant extracts (pomegranate (Punica Granatum), Eucalyptus, and pecan (Carya illinoinensis, leaves), metal species (copper and iron), metallic concentrations, and type of functionalization $(\mathrm{OH}$ and $\mathrm{COOH})$ on the characteristics of the obtained materials were studied. The precursor and impregnated MWCNTs were characterized through X-ray diffraction, Fourier transformed infrared spectroscopy, scanning electron microscopy, energy-dispersive X-ray spectroscopy, point of charge, N2 adsorption/desorption isotherms and, X-ray photoelectron spectroscopy. All the synthesized materials were tested as adsorbents to remove glyphosate (GLY) in an aqueous medium. The MWCNTs were resistant to withstand the synthesis process, preserving its structure and morphological characteristics. The copper and iron on the surface of MWCNTS confirm the successful synthesis and impregnation of the MNPs. The MWCNTs impregnated with high metallic concentrations showed favorable adsorption of GLY. The adsorption capacity and percentage of removal were $21.17 \mathrm{mg} \mathrm{g-1}$ and $84.08 \%$, respectively, for the MWCNTs impregnated with iron MNPs using the pecan leaves as a reducing agent. The results indicated that an advanced adsorbent for GLY could be obtained by green synthesis, using MWCNTs as precursors and pecan leaves as a reducing agent.
\end{abstract}

\section{Keywords}

Glyphosate, Adsorption, Carbon nanotubes, Nanoparticles, Green synthesis, XPS analyses 